\title{
Baicalein inhibits tumor progression by inhibiting tumor cell growth and tumor angiogenesis
}

\author{
YANG-GYU PARK $^{1}$, JAWUN CHOI ${ }^{1}$, HYE-KANG JUNG ${ }^{1}$, BUMSEOK KIM ${ }^{1}$, \\ CHAN KIM $^{2}$, SANG-YOUEL PARK ${ }^{1}$ and JAE-WON SEOL ${ }^{1}$ \\ ${ }^{1}$ Biosafety Research Institute, College of Veterinary Medicine, Chonbuk National University, Iksana; \\ ${ }^{2}$ CHA Bundang Medical Center, CHA University, Seongnam, Republic of Korea
}

Received June 23, 2017; Accepted September 18, 2017

DOI: $10.3892 /$ or.2017.6007

\begin{abstract}
Baicalein, a herbal medicine, is a natural flavonoid isolated from the roots of Scutellaria baicalensis Georgi. It is known for its anticancer, anti-inflammatory and neuroprotective properties. Despite these well-known properties, it is not yet clear what effect baicalein has on tumor progression. Therefore, in the present study, we used B16F10 cells, Lewis lung carcinoma (LLC) cells, and human umbilical vein endothelial cells (HUVECs) to investigate the effect of baicalein on cell proliferation and viability, migration and tube formation in vitro. In addition, an experimental animal model was used to observe the growth rate and metastasis of tumors and tumor vessel formation in vivo. Our results showed that baicalein decreased the proliferation and migration and induced tumor cell death via caspase-3 activation in the B16F10 and LLC cells, and strongly inhibited tube formation and cell migration in HUVECs. Furthermore, mouse models showed that baicalein reduced the tumor volume and greatly reduced the tumor growth rate in the early stages of tumor progression, and the baicalein-treated groups had significantly reduced expression of CD31 (endothelial cell marker) and $\alpha$-SMA (mural cell marker) in the tumors, indicating that baicalein inhibits tumor angiogenesis by disrupting tumor vasculature development. Comparison of the lymph node and lung samples collected from the baicalein-treated group, and the untreated group showed that baicalein reduced metastasis of the tumor to these tissues. In summary, baicalein reduced tumor progression and metastasis, directly induced tumor cell death, and inhibited tumor angiogenesis. Our results strongly demonstrate that baicalein is a potential chemotherapeutic agent.
\end{abstract}

Correspondence to: Professors Sang-Youe Park or Jae-Won Seol, Biosafety Research Institute, College of Veterinary Medicine, Chonbuk National University, Iksan-si, Jeollabuk-do 54596, Republic of Korea

E-mail: sypark@jbnu.ac.kr

E-mail: jwsseol@jbnu.ac.kr

Key words: baicalein, cancer, metastasis, caspase-3, angiogenesis

\section{Introduction}

A solid tumor is an abnormal mass of tissue (excluding hematological malignancies) and can be classified as benign (non-cancerous) and malignant (cancerous). Solid tumors are classified according to the type of cells forming the tumor, where localized solid tumors are either sarcoma, carcinoma, or lymphoma (1). Solid tumor cancers directly affect patient survival, particularly when tumors are malignant (2). Improving the survival rate of cancer patients requires suppression of the development and growth of cancer (3). Although most tumor treatments include surgery, radiation therapy and chemotherapy, these methods are limited by tumor metastasis (4). Therefore, it is necessary to develop chemotherapeutic methods using innocuous agents to complement existing therapies.

Baicalein is a herbal medicine that has long been used as a chemotherapeutic agent and an anti-inflammatory agent $(5,6)$. It is a flavonoid isolated from the roots of S. baicalensis Georgi and is known to function by inducing apoptosis, initiating autophagy, and causing cell cycle arrest (7-10). Recent studies have shown that baicalein inhibits carcinoma metastasis through inhibition of cell motility and cell migration (11).

Tumor angiogenesis is necessary for tumor progression, as the vascular network supplies oxygen and nutrients and removes waste products $(12,13)$. Therefore, tumor growth and metastasis are dependent on angiogenesis and lymphangiogenesis (14-16). Recently, several studies found that baicalein inhibited proliferation of smooth muscle cells and inhibited LPS-induced angiogenesis in vascular endothelial cells $(6,17)$. Baicalein was also found to inhibit endothelial cell proliferation by inhibiting VEGFR-2 phosphorylation and to decrease the activity of MMP-2 in endothelial cells $(18,19)$. The anticancer effect of baicalein is mostly related to ERK signaling and MMP-2 activation $(7,11,20)$.

Previous studies have indicated that the anticancer effect of baicalein may result from simultaneous action on tumor cells and vascular endothelial cells. In the present study, we used B16F10 cells, Lewis lung carcinoma (LLC) cells, and human umbilical vein endothelial cells (HUVECs) to investigate the effect of baicalein on these cells in vitro. In addition, an experimental animal model was used to observe the growth rate and metastasis of tumors and tumor vessel formation 
in vivo. Our results confirmed that baicalein decreased both tumor metastasis and the tumor growth rate, and inhibition of tumor growth was mediated by baicalein-induced tumor cell death and suppression of vasculature formation in the tumor. The purpose of the present study was to investigate the effect of baicalein on tumor cells and its impact on the formation of the tumor vascular network in order to assess its potential as a safe and effective anticancer agent.

\section{Materials and methods}

Mice. All animal experiments were performed with approval from the Animal Care Committees of Chonbuk National University. Specific pathogen-free C57BL/6 mice were purchased from the Samtako Bio Korea Co., Ltd. (Osan, Korea). To confirm both the effects on tumor cells and tumor angiogenesis, C57BL/6 mice were used in the experiment based on a previous study (12). All mice were transferred to our pathogen-free animal facilities and given ad libitum access to a standard diet (PMI LabDiet) and water. Mice used for the present study were 7 weeks old. All mice were divided into 4 groups: B16F10 injected, baicalein-treated after B16F10 injection, LLC injected and baicalein treated after LLC injection.

Tumor model and baicalein treatment. B16F10 melanoma cells were obtained from the Korean Cell Line Bank and Lewis lung carcinoma LLC cells were obtained from Bundang CHA Medical Center. To generate the tumor models, suspended tumor cells $\left(1 \times 10^{6}\right.$ cells in $\left.100 \mu \mathrm{l}\right)$ were subcutaneously (s.c.) injected into the dorsal flank of 7-week-old male mice. After implantation, $1.5 \mathrm{mg} / \mathrm{kg}$ baicalein was intraperitoneally (i.p.) injected at the same time every day for 2 weeks from day 7 after injection of the tumor cells. Tumor volume and tumor growth rate were measured using previously reported methods (12).

Histological analysis. The mice were sacrificed by cervical dislocation on the indicated days. Tumor tissues and lymph nodes were fixed in $4 \%$ paraformaldehyde (PFA) for $4 \mathrm{~h}$, dehydrated in $20 \%$ sucrose solution overnight, and embedded with tissue freezing medium (Leica, Wetzlar, Germany). Frozen blocks were cut into $80-\mu \mathrm{m}$ sections. Samples were blocked with 5\% donkey (or goat) serum in PBS-Tween-20 (PBST) $(0.03 \%$ Triton X-100 in PBS), and then incubated for $4 \mathrm{~h}$ at room temperature (RT; range, $20-27^{\circ} \mathrm{C}$ ) with the following primary antibodies: anti-CD31 (hamster, clone 2H8; Millipore Billerica, MA, USA), FITC-conjugated anti- $\alpha$-SMA (mouse, clone 1A4; Sigma-Aldrich, St. Louis, MO, USA), anti-caspase-3 (rabbit polyclonal; R\&D Systems, Minneapolis, MN, USA), anti-LYVE-1 (rabbit polyclonal; AngioBio, Del Mar, CA, USA), and anti-pan-cytokeratin (mouse, clone AE1/AE3; Abcam, Cambridge, MA, USA). After 3-5 washes, the samples were incubated for $2 \mathrm{~h}$ at RT with the following secondary antibodies: Cy3-conjugated antihamster IgG (127-165-160), Cy3-conjugated anti-mouse IgG (715-165-150), Cy3-conjugated anti-rabbit IgG (711-165-152) and FITC-conjugated anti-rabbit IgG (711-095-152) (Jackson ImmunoResearch, West Grove, PA, USA). Nuclei were stained with 4',6-diamidino-2-phenylindole (DAPI). The samples were then mounted in fluorescent mounting medium (Dako, Carpinteria, CA, USA) and immunofluorescent images were acquired using a Zeiss LSM 510 confocal fluorescence microscope (Carl Zeiss, Jena, Germany).

For hematoxylin and eosin (H\&E) staining, lung tissues were fixed overnight in 4\% PFA. After tissue processing using standard procedures, samples were embedded in paraffin and cut into $5-\mu \mathrm{m}$ sections followed by $\mathrm{H} \& \mathrm{E}$ staining.

Morphometric analysis. Blood vessel density measurements in the intratumoral regions were analyzed using photographic analysis in the ImageJ software (http://rsb.info.nih.gov/ij) and the LSM Image Browser (Carl Zeiss). For blood vessel density, the CD31-positive area (per random $0.44 \mathrm{~mm}^{2}$ area) was measured in the intratumoral region. The range of $\alpha$-SMA-positive mural cells was calculated as a percentage of the corresponding positive fluorescence region along the CD31-positive blood vessels in random $0.44 \mathrm{~mm}^{2}$ intratumoral regions.

Cell culture and reagents. HUVEC growth medium was purchased from Lonza Group Ltd. (Basel, Switzerland). Cells were cultured in endothelial cell growth medium (EGM-2 MV BulletKit) and used at passage 3-4 in all experiments. B16F10 and LLC cells were cultured in Dulbecco's modified Eagle's medium (DMEM) (purchased from Gibco, Grand Island, NY, USA) supplemented with 10\% FBS (purchased from Atlas Biologicals, Fort Collins, CO, USA), $100 \mathrm{U} / \mathrm{ml}$ penicillin and $100 \mu \mathrm{g}$ streptomycin (purchased from Sigma). All cells were incubated at $37^{\circ} \mathrm{C}$ with $5 \% \mathrm{CO}_{2}$. Baicalein (purchased from Sigma) was dissolved in dimethyl sulfoxide (DMSO) to create a stock solution at $100 \mathrm{mM}$ (for in vitro use) or $3 \mathrm{mg} / \mathrm{ml}$ (for in vivo use), which was then diluted with autoclaved PBS buffer prior to use.

Cell proliferation and cell viability assay. Cell proliferation was detected by MTT assay and cell viability was detected by Annexin V assay. The colorimetric 3-4,5-dimethylthiazol-2-yl-2,5-diphenyl tetrazolium bromide (MTT) assay was performed to quantitate the effect of baicalein on mitochondrial dysfunction. Briefly, $1 \times 10^{5}$ cells/well were seeded in a 24-well microplate in a final volume of $300 \mu$ l, incubated overnight, and treated with baicalein for $24 \mathrm{~h}$. Following treatment for $24 \mathrm{~h}, 30 \mu \mathrm{l}$ of MTT ( $5 \mathrm{mg} / \mathrm{ml}$ in PBS) was added to the cells and incubation was carried out for $2 \mathrm{~h}$. The MTT solution was then removed and replaced by $300 \mu \mathrm{l}$ dimethyl sulfoxide (DMSO), and the plates were shaken for $10 \mathrm{~min}$. Then, $200 \mu \mathrm{l}$ of the sample were transferred to a 96 -well microplate. The optical density was determined at a wavelength of $570 \mathrm{~nm}$. Apoptosis was assessed by a commercial Annexin V assay (Santa Cruz Biotechnology) according to the manufacturer's protocol. Annexin V content was determined by measuring fluorescence at excitation $488 \mathrm{~nm}$ and emission at $525 \mathrm{~nm}$ using a Guava EasyCyte HT system (Millipore).

Scratch wound healing assay. Tumor cells (B16F10, LLC) and HUVECs were grown on 6-well plates. Cultured cells were wounded by scraping with a $1,000 \mu 1$ pipette tip. After wounding, the cells were gently washed twice with PBS and incubated with $2 \%$ FBS-containing medium supplemented 
with $100 \mu \mathrm{M}$ baicalein for $24 \mathrm{~h}$. Then, the wounded areas were observed and photographed using a microscope (Nikon Eclipse TS100; Nikon Corporation, Tokyo, Japan) at a magnification of $\mathrm{x} 10$.

In vitro tube formation assay. Tube formation was assessed by an endothelial cell tube formation assay (Corning Inc., Corning, NY, USA) according to the manufacture's protocol. Tube number was counted in 3 random fields using ImageJ software (http://rsb.info.nih.gov/ij).

Western blotting. B16F10 and LLC cells were homogenized in cold RIPA buffer containing a protease inhibitor cocktail $18 \mathrm{~h}$ after baicalein treatment. Each protein was separated by SDS-PAGE gel and transferred to nitrocellulose membranes. After blocking with 5\% skim milk, the membranes were incubated with anti-caspase-3 (rabbit monoclonal) and anti-cleaved caspase-3 (rabbit) and anti-PARP (rabbit) (all from Cell Signaling Technology, Inc., Beverly, MA, USA) and anti- $\beta$-actin antibody (mouse monoclonal; SigmaAldrich) in blocking buffer overnight at $4^{\circ} \mathrm{C}$, and then with HRP-conjugated secondary antibody for $2 \mathrm{~h}$ at RT. Signals were developed with enhanced chemiluminescence HRP substrate (Millipore) and detected using the Fusion FX7 acquisition system (Vilbert Lourmat, Eberhardzell, Germany).

Immunocytochemical analysis. Tumor cells (B16F10, LLC) were cultured on glass slides, fixed with cold acetone and blocked using 5\% donkey serum in Tris-buffered saline with Tween-2 (TBST). The cells were incubated with anticaspase-3 (rabbit polyclonal; R\&D Systems) at $4^{\circ} \mathrm{C}$. Cells were incubated with Cy3-conjugated anti-rabbit IgG (Jackson ImmunoResearch, 711-165-152). Nuclei were stained with DAPI. Then, the samples were mounted in fluorescent mounting medium (Dako) and immunofluorescent images were acquired using a Zeiss LSM510 confocal fluorescence microscope (Carl Zeiss).

Statistical analysis. Values are presented as mean \pm standard deviation (SD). Significant differences between means were determined by unpaired Student t-tests or analysis of variance with one-way ANOVA followed by the Student-Newman-Keuls test. Statistical significance was set as $<0.05$ and indicated as ${ }^{*} \mathrm{p}<0.05$ or ${ }^{* *} \mathrm{P}<0.01$ in the figures and legends.

\section{Results}

Baicalein inhibits tumor growth via activation of caspase-3. We administered baicalein to mice injected with B16F10 melanoma or LLC carcinoma cells in order to examine its anticancer effects on the two solid tumor models. As a result of the baicalein treatment, tumor size and tumor volume were reduced in each model compared to the untreated group (Fig. 1A and B). The maximum effect of baicalein treatment was noted on day 11 (49.20\% inhibition) for the B16F10-implanted group and day 19 (48.25\% inhibition) for the LLC-implanted group (Fig. 1C). Notably, the tumor growth rate was significantly reduced in the baicalein-treated group compared to the untreated group, with the most significant decrease observed 11 days after tumor implantation (Fig. 1D and E). As shown in Fig. 1D and E, tumor growth rates in the B16F10- and LLC-injected mice decreased by 144 and $51 \%$, respectively, upon treatment with baicalein. In vitro measurements of cell proliferation with B16F10 and LLC cells revealed dose-dependent decreases by baicalein treatment (Fig. 2). Inhibition of cell proliferation by baicalein was greater in the B16F10 cells than that in the LLC cells at a given treatment concentration. Viability of B16F10 and LLC cells was decreased upon baicalein treatment by 35 and $22 \%$, respectively. In addition, baicalein strongly inhibited tumor cell migration (Fig. 2C and D). The Annexin $\mathrm{V}$ assay was performed to assess whether baicalein induces apoptosis of tumor cells. The results showed that cell viability was reduced by $55 \%$ in the B16F10 cells and $31 \%$ in the LLC cells (Fig. 3A). Western blot methods were used to assess the effect of baicalein on the expression and activation of caspase-3 (Cas3). Although Cas3 expression was not significantly changed, Cas3 activation (cleaved-cas3) through proteolytic cleavage was increased in a dose-dependent manner after baicalein treatment (Fig. 3B). In addition, Cas3 activation through cleavage was confirmed via immunofluorescence staining in both in vitro tumor cells and in vivo tumor tissue samples (Fig. 3C and D). To determine whether apoptosis by baicalein was dependent on Cas3 activation, we confirmed the expression of PARP. As a result, cleaved-PARP was also increased by baicalein treatment (Fig. 3E). Therefore, these results demonstrated that baicalein treatment induced apoptosis via Cas3 activation.

Baicalein inhibits vasculature formation by directly affecting endothelial cells during tumor angiogenesis. Tumor tissue was stained with CD31 (endothelial cell specific) and $\alpha$-SMA (mural cell specific) immunofluorescent markers to confirm the effect of baicalein on tumor angiogenesis (Fig. 4A and B). The density of blood vessels was markedly reduced by baicalein treatment in the tumor models with B16F10 and LLC implanted cells. In mice injected with B16F10 cells, intratumoral blood vessel density was $37.56 \%$ in the untreated group and $21.24 \%$ in the baicalein-treated group. The mice injected with LLC cells showed a pattern similar to the B16F10-injected group, with intratumoral blood vessel densities of $14.88 \%$ in the untreated group and $9.51 \%$ in the baicalein-treated group. $\alpha$-SMA was expressed at very low levels. These results indicate that baicalein treatment strongly inhibited vasculature formation during tumor progression. HUVECs were treated with baicalein and the effects on angiogenesis were examined in a tube formation assay and a cell migration assay (Fig. 4C and D). Baicalein strongly suppressed tube formation by almost $80 \%$ or more at concentrations above $100 \mu \mathrm{M}$. A cell migration assay with $100 \mu \mathrm{M}$ baicalein significantly reduced migration of HUVECs. These results suggest that baicalein directly affects vascular endothelial cells to suppress angiogenesis.

Baicalein delays tumor metastasis in the LLC model. Our results showed that baicalein inhibited tumor angiogenesis, while tumor cells are known to spread to other organs via the tumor vasculature. Therefore, we used LLC-injected mouse models to investigate the antimetastatic effect of baicalein treatment. To confirm the effect of baicalein on metastasis, we separated the inguinal lymph nodes and lungs of the LLC-implanted mice and compared the control and 
A

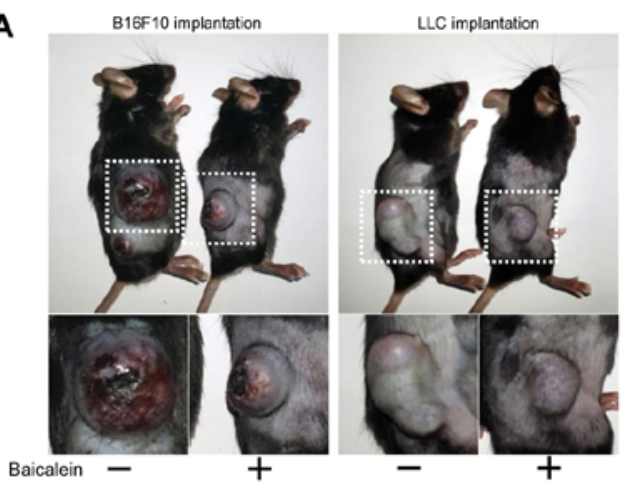

B

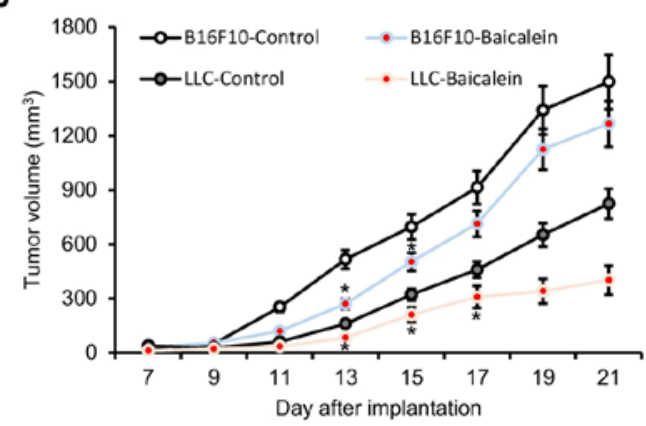

C

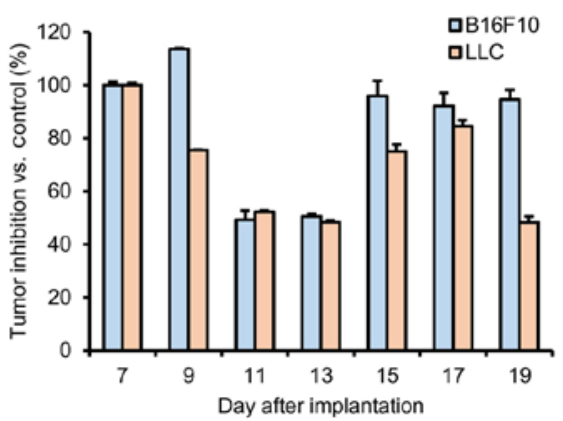

D

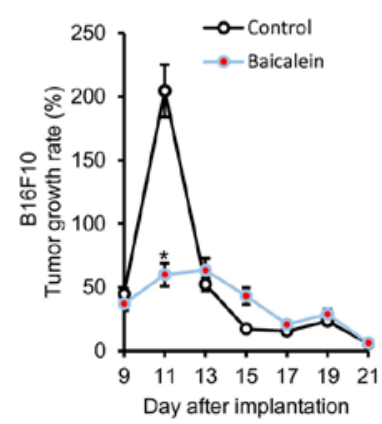

E

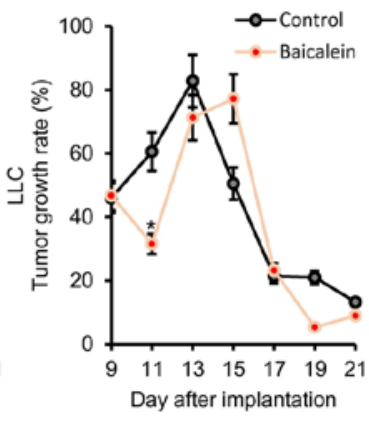

Figure 1. Baicalein inhibits tumor progression in mouse tumor models. (A) The image shows tumor development 21 days after implantation of B10F10 and LLC cells. The magnified images compare the baicalein-treated and untreated group in the mouse tumor models (each group, $n=15$ ). (B) A comparison of the tumor volume between the baicalein-treated and the untreated group in the tumor-implanted mice (each group, $n=6$ ). (C) The percentage of inhibition of tumor volume as determined in B. (D and E) A comparison of the tumor growth rate between the baicalein-treated and the untreated group in the B10F10-implanted and LLC-implanted mice (each group, $\mathrm{n}=6$ ). Fifteen mice/group, of which 6 randomly selected mice were used for tumor volume and tumor growth rate comparisons. Data are presented as the mean \pm standard deviations from more than 3 independent experiments; ${ }^{*} \mathrm{P}<0.05$.

A

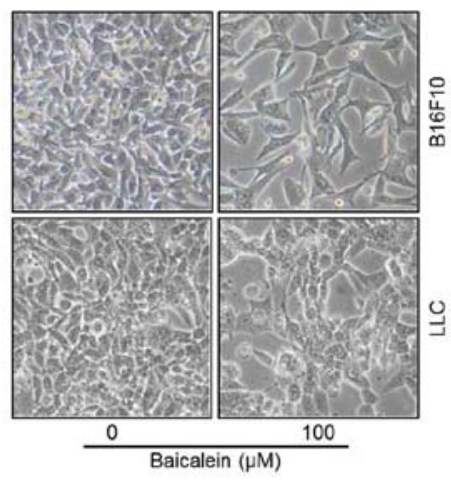

C

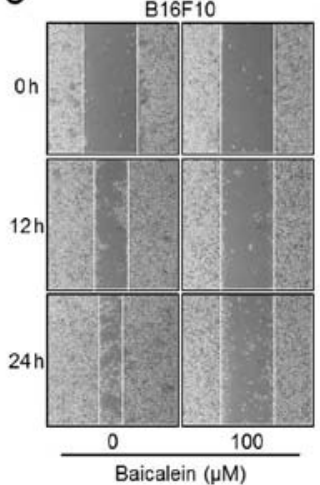

B

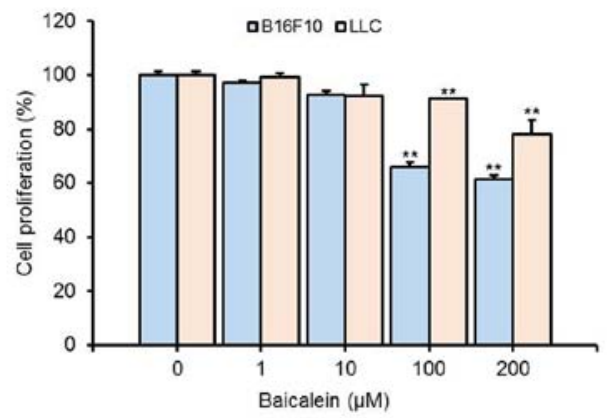

D

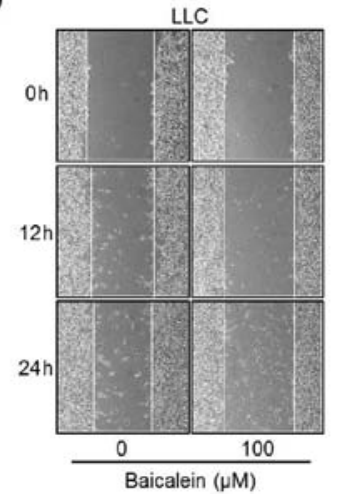

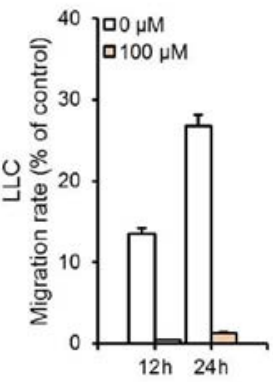

Figure 2. Baicalein inhibits the cell proliferation and migration in B16F10 and LLC cells. (A) Morphology of the tumor cells (B16F10 and LLC) after $24 \mathrm{~h}$ in the baicalein-treated and untreated groups (magnification, x100). (B) Tumor cells (B16F10 and LLC) were treated with baicalein for $24 \mathrm{~h}$, and then, cell proliferation was measured using the MTT assay. Data are presented as the mean \pm standard deviations from more than 3 independent experiments; ${ }^{* *} \mathrm{P}<0.01$. $(\mathrm{C}$ and $\mathrm{D})$ The scratch wound healing assay was used to evaluate the motility of B16F10 and LLC cells in the baicalein-treated and untreated groups with assessments $24 \mathrm{~h}$ after treatment. The percent migration rate is expressed as a percentage of the untreated group. 
A

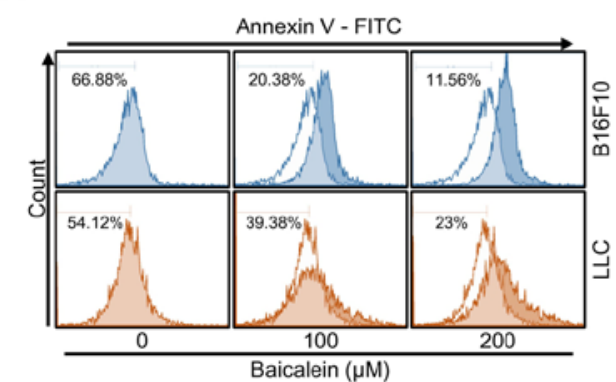

C

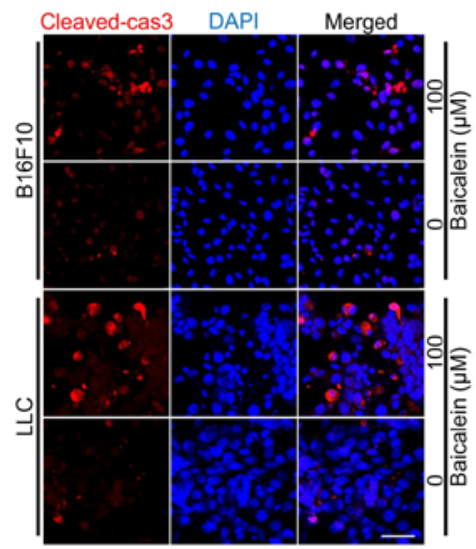

B

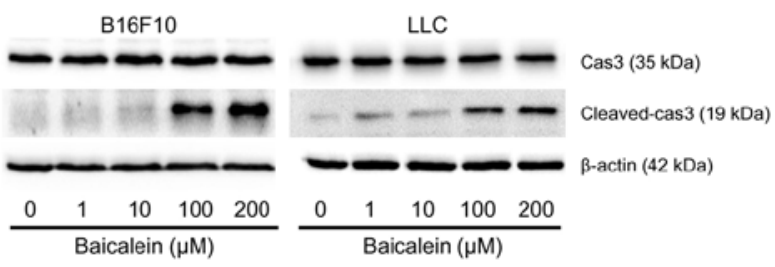

E

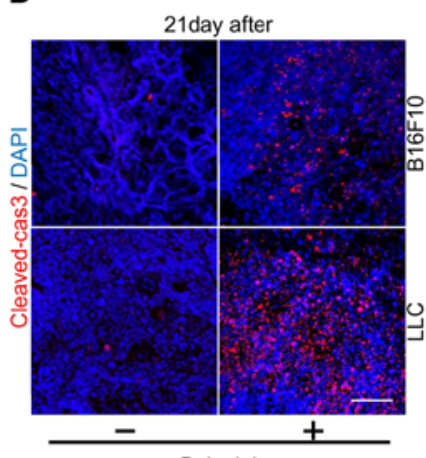

B16F10

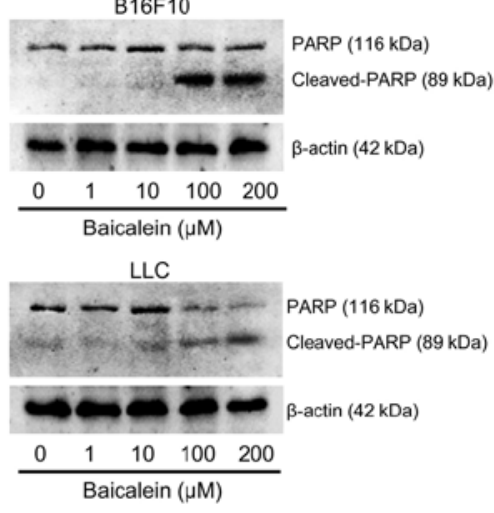

Figure 3. Baicalein induces tumor cell death via activation of caspase-3. (A) Tumor cells (B16F10 and LLC) were treated with baicalein for $24 \mathrm{~h}$, and then, cell viability was measured with the Annexin V assay. (B) Caspase-3 protein expression [both activated (cleaved-cas3) and total forms (Cas3)] was assessed in the B16F10 and LLC cells by western blotting with samples collected $18 \mathrm{~h}$ after baicalein treatment. (C) Immunocytochemistry was performed on B16F10 and LLC cells $24 \mathrm{~h}$ after baicalein treatment to determine cleaved-cas3 protein levels (scale bars, $50 \mu \mathrm{m}$ ). The results presented are representative of the observations. (D) Immunohistochemistry was performed on tumor tissue 21 days after tumor implantation to determine cleaved-cas 3 protein levels (scale bars, $100 \mu \mathrm{m}$ ). The results presented are representative of the observations. (E) PARP expression [both activated (cleaved-PARP) and total forms (PARP)] was assessed in B16F10 and LLC cells by western blotting with samples collected $18 \mathrm{~h}$ after baicalein treatment.

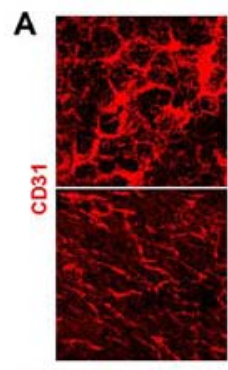

Baicalein -

C

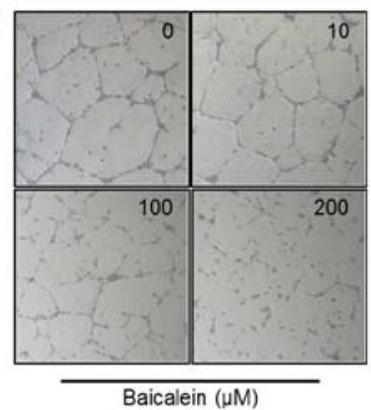

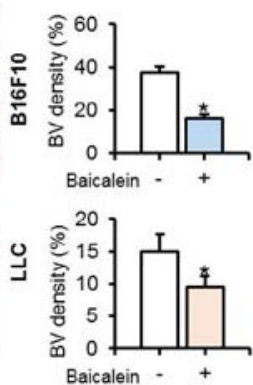

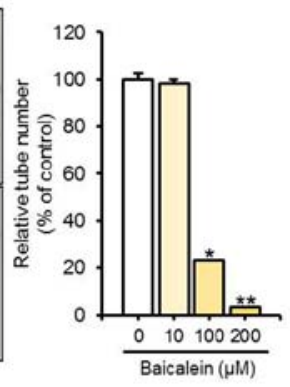

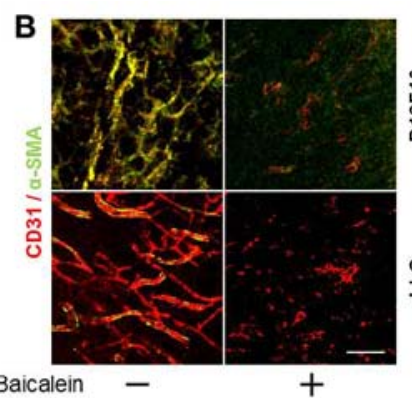
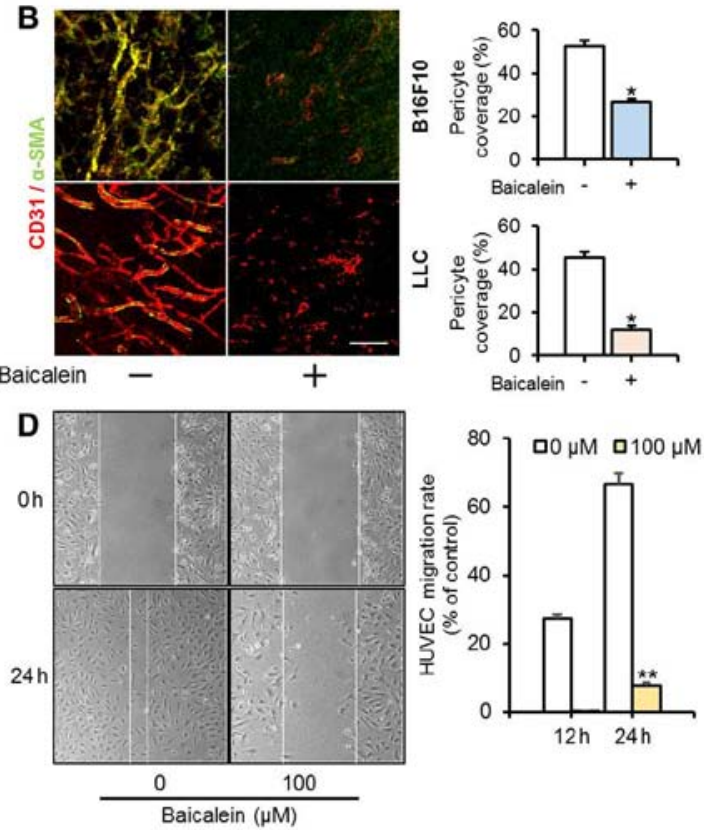

Figure 4. Baicalein inhibits tumor growth through inhibition of tumor angiogenesis. (A) A comparison of CD31-positive tumor vessels in baicalein-treated $(1.5 \mathrm{mg} / \mathrm{kg}$, daily) and untreated mice carrying either B16F10 or LLC cell tumors (14 days of baicalein exposure). Blood vessel density is expressed as a percentage of the untreated group (scale bars, $100 \mu \mathrm{m}$ ). (B) A comparison of $\alpha$-SMA expression (in CD31-positive tumor vessels) in baicalein-treated and untreated mice carrying either B16F10 or LLC cell tumors (14 days of baicalein exposure). Pericyte coverage is expressed as a percentage of the untreated group (scale bars, $100 \mu \mathrm{m}$ ). (C) Tube formation of HUVECs on Matrigel induced by baicalein treatment (18 h). Tube number is expressed as a percentage of the untreated group. (D) The scratch wound healing assay was used to evaluate motility of HUVECs in baicalein-treated and untreated groups after 24 h. The percent migration rate is expressed as a percentage of the untreated group. Data are presented as the mean \pm standard deviations from $\geq 3$ independent experiments; ${ }^{*} \mathrm{P}<0.05$ or ${ }^{* *} \mathrm{P}<0.01$. 
A

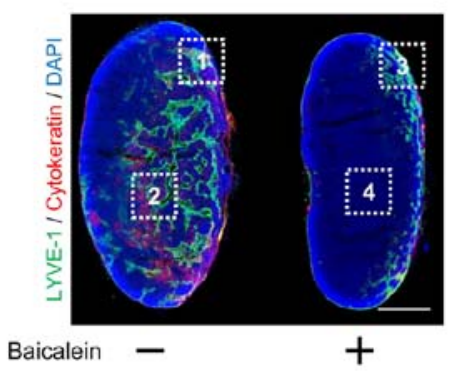

C

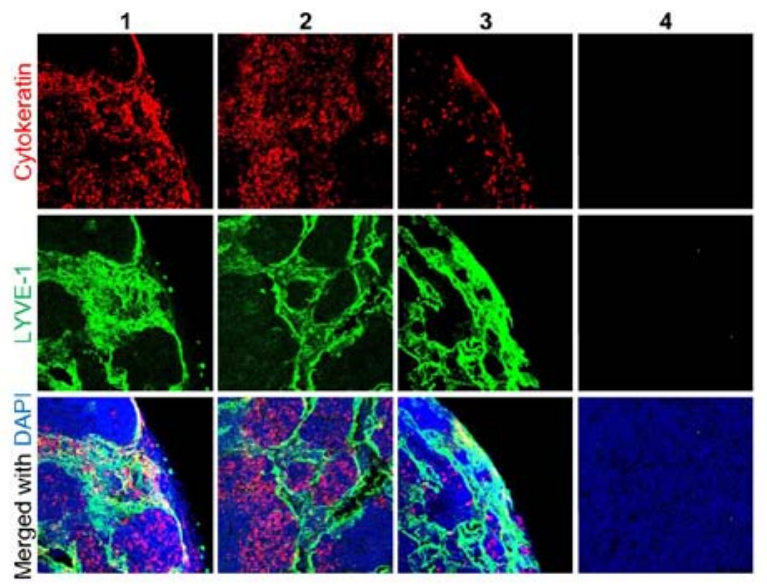

B

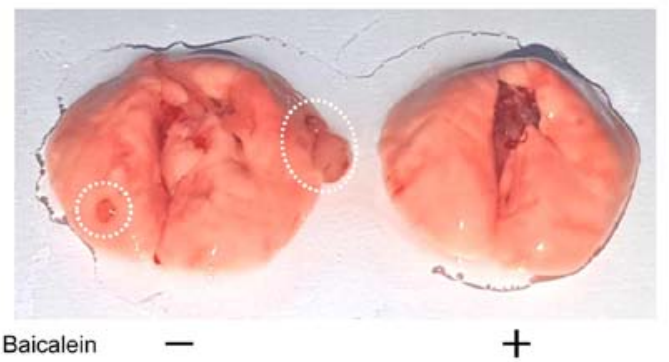

D

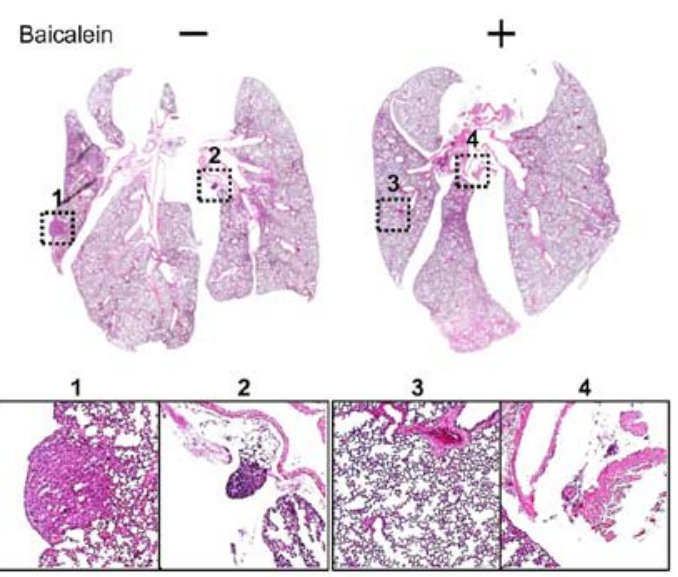

Figure 5. Baicalein delays tumor metastasis in the LLC model. (A and C) Cytokeratin-positive tumor cells are shown (red) metastasized to inguinal lymph nodes. (B) The morphology of lungs are shown in baicalein-treated mice and untreated mice 21 days after tumor implantation. (D) Lung samples were stained with H\&E and metastatic regions are shown as a magnified image. Magnified images show the metastatic region in the LLC-implanted tumors of the baicaleintreated and untreated groups.

Table I. Effect of baicalein on tumor and endothelial cells.

\begin{tabular}{|c|c|c|c|c|c|c|c|c|}
\hline \multirow[b]{3}{*}{ Type of cells } & \multicolumn{2}{|c|}{$\begin{array}{c}\text { Cell proliferation } \\
(\%)\end{array}$} & \multicolumn{4}{|c|}{$\begin{array}{l}\text { Cell migration rate } \\
\text { (\% of control) }\end{array}$} & \multicolumn{2}{|c|}{$\begin{array}{l}\text { Relative tube number } \\
\text { (\% of control) }\end{array}$} \\
\hline & \multirow[b]{2}{*}{ Control } & \multirow{2}{*}{$\begin{array}{l}\text { Baicalein } \\
(100 \mu \mathrm{M})\end{array}$} & \multicolumn{2}{|c|}{ Control } & \multicolumn{2}{|c|}{ Baicalein $(100 \mu \mathrm{M})$} & \multirow[b]{2}{*}{ Control } & \multirow{2}{*}{$\begin{array}{l}\text { Baicalein } \\
(100 \mu \mathrm{M})\end{array}$} \\
\hline & & & $12 \mathrm{~h}$ & $24 \mathrm{~h}$ & $12 \mathrm{~h}$ & $24 \mathrm{~h}$ & & \\
\hline B16F10 & 100 & $65.86 \pm 1.66^{\mathrm{b}}$ & $31.40 \pm 2.01$ & $56.81 \pm 2.57$ & $4.28 \pm 1.82$ & $6.63 \pm 1.99^{\mathrm{b}}$ & - & - \\
\hline LLC & 100 & $91.24 \pm 0.03^{\mathrm{b}}$ & $13.46 \pm 0.72$ & $26.8 \pm 1.34$ & $0.42 \pm 0.02$ & $1.24 \pm 0.05^{\mathrm{b}}$ & - & - \\
\hline HUVECs & - & - & $27.18 \pm 1.45$ & $66.59 \pm 3.48$ & $0.23 \pm 0.01$ & $7.74 \pm 0.43^{b}$ & $99.99 \pm 2.75$ & $23.21 \pm 1.59^{\mathrm{a}}$ \\
\hline
\end{tabular}

Data are expressed as mean \pm SD from 3 independent experiments; ${ }^{\mathrm{a}} \mathrm{P}<0.05,{ }^{\mathrm{b}} \mathrm{P}<0.01$ as compared to the control. LLC, Lewis lung carcinoma; HUVECs, human umbilical vein endothelial cells.

baicalein treatment groups. As a result, baicalein was found to delay tumor metastasis. In addition, the lymph node of the baicalein-treated group showed a similar size to the wild-type lymph node. As shown in Fig. 5A and C, the lymph nodes of the baicalein-treated mice were smaller than those of the untreated mice, in which the lymph nodes were hypertrophic. We determined the LLC cells within the lymph nodes by immunohistochemistry using the cytokeratin (LLC marker) and LYVE-1 (lymphatic marker) antibodies. These experiments also showed that baicalein reduced LLC metastasis to the lymph node (Fig. 5A and C). In addition, it was confirmed that the LLC metastasis to the lungs was reduced by baicalein treatment (Fig. 5B and D). These results showed that baicalein can attenuate tumor cell metastasis by inhibiting formation of the intratumoral vasculature.

\section{Discussion}

Baicalein is a natural flavonoid isolated from the root of S.baicalensis Georgi that has long been used as a chemotherapeutic agent due to its anticancer and anti-inflammatory properties (7-9). Recent studies have also shown that baicalein 
inhibits both mural cell proliferation and LPS-induced angiogenesis in vascular endothelial cells (6). Despite considerable research on baicalein, its effects on tumor progression and metastasis are not yet clear. In the present study, we demonstrated the anticancer effect of baicalein on the progression of tumors such as melanoma and carcinoma. In order to elucidate the anticancer effect of baicalein, we focused on the following 3 points: first, the effects on tumor models and cells; second, the effects on the development of tumor vasculature; and third, the effects on tumor metastasis.

Our results showed that the tumor growth rate was strongly inhibited by baicalein (Fig. 1) and tumor development was suppressed in the early stages of tumor progression. As shown in Fig. 1D and E, after 11 days of tumor implantation, tumor growth rates were decreased in both the control and baicalein treatment groups. However, in the treated group with baicalein at the early stage of tumor progression, the tumor growth rates were relatively decreased compared to the control group. During tumor progression, tumor cells in the intratumoral core of the tumor tissues exhibit slow proliferation, and growth rates are small (21). Nevertheless, on day 11 after tumor implantation, tumor growth rates were markedly decreased by baicalein treatment. However, the tumor growth rates after day 11 were similar in both the control and treatment groups. These results indicated that the anticancer effect of baicalein is strong at the early stage of tumor progression.

Previous studies have shown that baicalein induces cell cycle arrest, leading to anticancer effects $(7,20)$. The anticancer effect of baicalein is thought to inhibit tumor cell proliferation and induce apoptosis, thereby inhibiting tumor cell progression $(22,23)$. We examined the anticancer effect of baicalein in B16F10 and LLC cells. Baicalein inhibited the proliferation and migration of tumor cells, and although the expression of caspase 3 was barely altered by baicalein treatment, caspase 3 activation through proteolytic cleavage was markedly increased (Fig. 3). These results demonstrate that baicalein increased caspase 3 and PARP activation, which induced apoptosis. Activation of caspase 3 was also observed in tumor tissues in vivo, consistent with the in vitro experiments (Fig. 3C and D).

Tumor angiogenesis is an essential process for tumor development and tumor cells utilize the tumor vasculature to metastasize to other tissues $(12,13)$. Baicalein was found to inhibit the proliferation of vascular smooth muscle cells (VSMCs) in cardiovascular disease (24). Notably, baicalein inhibited the expression of VEGF and FGFR-2, leading to anticancer effects in lung cancer (20). As the expression of VEGF and VSMCs are closely related to angiogenesis $(25,26)$, we expected that baicalein may affect the process of vasculature formation in tumors. We confirmed that baicalein inhibited the expression of CD31 and $\alpha$-SMA in tumor tissues and strongly inhibited tube formation and cell migration in vascular endothelial cells (HUVECs). The antimetastatic effect of baicalein was verified in the lymph node and lung concurrently with observations of tumor vasculature formation. Baicalein-mediated disruption of tumor angiogenesis produced an expected delay in tumor metastasis to both the lymph nodes and the lungs, as the tumor vasculature provides one of the metastatic routes. These results indicate that baicalein affects both tumor cells and vascular endothelial cells in the tumor during tumor progression. Notably, the anticancer effects of baicalein on tumor cells were more strongly detected in B16F10 cells than LLC cells. However, the in vivo experiment showed stronger anticancer effects in the LLC-implanted mice. In addition, the tumor volume of LLC-implanted mice was relatively smaller than that of the B16F10-implanted mice. These results suggest that the anticancer effect of baicalein was not efficiently acting on the tumor cells in the intratumoral core of the tumor mass during tumor progression. To summarize our results, the therapeutic effects of baicalein not only affect tumor mass but also tumor angiogenesis (Table I).

Taken together, our results demonstrated the anticancer effect of baicalein. Firstly, baicalein inhibited tumor cell proliferation and induced apoptosis. Secondly, baicalein inhibited tumor progression by disrupting intratumoral vasculature formation. Thirdly, baicalein delayed tumor metastasis. Our results strongly suggest that baicalein is a useful chemotherapeutic agent, owing to its multifunctional effects on tumor cells and vascular endothelial cells during tumor progression. Based on the present study, we aim to identify the mechanisms related to baicalein-induced tumor growth inhibition in future research.

\section{Acknowledgements}

The present study was supported by the Co-operative Research Program for Agriculture, Science and Technology Development (PJ012612012017) in the Rural Development Administration and by the Korea Institute of Planning and Evaluation for Technology in Food, Agriculture, Forestry and Fisheries (IPET) through Agriculture, Food and Rural Affairs Research Center Support Program, funded by the Ministry of Agriculture, Food and Rural Affairs (MAFRA; 716002-7).

\section{References}

1. O'Neill AC, Jagannathan JP and Ramaiya NH: Evolving cancer classification in the era of personalized medicine: A primer for radiologists. Korean J Radiol 18: 6-17, 2017.

2. Lechner MG, Karimi SS, Barry-Holson K, Angell TE, Murphy KA, Church CH, Ohlfest JR, Hu P and Epstein AL: Immunogenicity of murine solid tumor models as a defining feature of in vivo behavior and response to immunotherapy. J Immunother 36: 477-489, 2013.

3. Miller KD, Siegel RL, Lin CC, Mariotto AB, Kramer JL, Rowland JH, Stein KD, Alteri R and Jemal A: Cancer treatment and survivorship statistics, 2016. CA Cancer J Clin 66: 271-289, 2016.

4. Rolston KVI: Infections in cancer patients with solid tumors: A review. Infect Dis Ther 6: 69-83, 2017.

5. Liu H, Dong Y, Gao Y, Du Z, Wang Y, Cheng P, Chen A and Huang H: The fascinating effects of baicalein on cancer: A review. Int J Mol Sci 17: 17, 2016.

6. Lee W, Ku SK and Bae JS: Anti-inflammatory effects of baicalin, baicalein, and wogonin in vitro and in vivo. Inflammation 38: 110-125, 2015.

7. Zhang Y, Song L, Cai L, Wei R, Hu H and Jin W: Effects of baicalein on apoptosis, cell cycle arrest, migration and invasion of osteosarcoma cells. Food Chem Toxicol 53: 325-333, 2013

8. Chen YJ, Wu CS, Shieh JJ, Wu JH, Chen HY, Chung TW, Chen YK and Lin CC: Baicalein triggers mitochondria-mediated apoptosis and enhances the antileukemic effect of vincristine in childhood acute lymphoblastic leukemia CCRF-CEM cells. Evid Based Complement Alternat Med 2013: 124747, 2013.

9. Wang Z, Jiang C, Chen W, Zhang G, Luo D, Cao Y, Wu J, Ding Y and Liu B: Baicalein induces apoptosis and autophagy via endoplasmic reticulum stress in hepatocellular carcinoma cells. Biomed Res Int 2014: 732516, 2014. 
10. Wang YF, Li T, Tang ZH, Chang LL, Zhu H, Chen XP, Wang YT and Lu JJ: Baicalein triggers autophagy and inhibits the protein kinase $\mathrm{B} / \mathrm{mammalian}$ target of rapamycin pathway in hepatocellular carcinoma HepG2 cells. Phytother Res 29: 674-679, 2015.

11. Chen K, Zhang S, Ji Y, Li J, An P, Ren H, Liang R, Yang J and Li Z: Baicalein inhibits the invasion and metastatic capabilities of hepatocellular carcinoma cells via down-regulation of the ERK pathway. PLoS One 8: e72927, 2013.

12. Kim C, Yang H, Fukushima Y, Saw PE, Lee J, Park JS, Park I, Jung J, Kataoka H, Lee D, et al: Vascular RhoJ is an effective and selective target for tumor angiogenesis and vascular disruption. Cancer Cell 25: 102-117, 2014.

13. Hanahan D and Weinberg RA: Hallmarks of cancer: The next generation. Cell 144: 646-674, 2011

14. Sundar SS and Ganesan TS: Role of lymphangiogenesis in cancer. J Clin Oncol 25: 4298-4307, 2007.

15. Gomes FG, Nedel F, Alves AM, Nör JE and Tarquinio SB Tumor angiogenesis and lymphangiogenesis: Tumor/endothelial crosstalk and cellular/microenvironmental signaling mechanisms. Life Sci 92: 101-107, 2013.

16. Foubert $P$ and Varner JA: Integrins in tumor angiogenesis and lymphangiogenesis. Methods Mol Biol 757: 471-486, 2012.

17. Dong LH, Wen JK, Miao SB, Jia Z, Hu HJ, Sun RH, Wu Y and Han M: Baicalin inhibits PDGF-BB-stimulated vascular smooth muscle cell proliferation through suppressing PDGFR beta-ERK signaling and increase in p27 accumulation and prevents injuryinduced neointimal hyperplasia. Cell Res 21: 1276-1276, 2011.

18. Ling Y, Chen Y, Chen P, Hui H, Song X, Lu Z, Li C, Lu N and Guo Q: Baicalein potently suppresses angiogenesis induced by vascular endothelial growth factor through the $\mathrm{p} 53 / \mathrm{Rb}$ signaling pathway leading to G1/S cell cycle arrest. Exp Biol Med 236: 851-858, 2011.
19. Huang Y, Miao Z, Hu Y, Yuan Y, Zhou Y, Wei L, Zhao K, Guo $\mathrm{Q}$ and $\mathrm{Lu} \mathrm{N}$ : Baicalein reduces angiogenesis in the inflammatory microenvironment via inhibiting the expression of AP-1. Oncotarget 8: 883-899, 2017.

20. Cathcart MC, Useckaite Z, Drakeford C, Semik V, Lysaght J, Gately K, O'Byrne KJ and Pidgeon GP: Anti-cancer effects of baicalein in non-small cell lung cancer in-vitro and in-vivo. BMC Cancer 16: 707, 2016.

21. Trédan O, Galmarini CM, Patel K and Tannock IF: Drug resistance and the solid tumor microenvironment. J Natl Cancer Inst 99: 1441-1454, 2007.

22. Yamashita S, Baba K, Makio A, Kumazoe M, Huang Y, Lin IC, Bae J, Murata M, Yamada S and Tachibana H: $\gamma$-Tocotrienol upregulates aryl hydrocarbon receptor expression and enhances the anticancer effect of baicalein. Biochem Biophys Res Commun 473: 801-807, 2016.

23. Liu TY, Gong W, Tan ZJ, Lu W, Wu XS, Weng H, Ding Q, Shu YJ, Bao RF, Cao Y, et al: Baicalein inhibits progression of gallbladder cancer cells by downregulating ZFX. PLoS One 10: e0114851, 2015.

24. Dong LH, Wen JK, Miao SB, Jia Z, Hu HJ, Sun RH, Wu Y and Han M: Baicalin inhibits PDGF-BB-stimulated vascular smooth muscle cell proliferation through suppressing PDGFR $\beta$-ERK signaling and increase in p27 accumulation and prevents injuryinduced neointimal hyperplasia. Cell Res 20: 1252-1262, 2010.

25. Cébe-Suarez S, Zehnder-Fjällman A and Ballmer-Hofer K: The role of VEGF receptors in angiogenesis; complex partnerships. Cell Mol Life Sci 63: 601-615, 2006.

26. Appelmann I, Liersch R, Kessler T, Mesters RM and Berdel WE: Angiogenesis inhibition in cancer therapy: platelet-derived growth factor (PDGF) and vascular endothelial growth factor (VEGF) and their receptors: biological functions and role in malignancy. Recent Results Cancer Res 180: 51-81, 2010. 